\title{
Einleitung: Kritisches Denken als gemeinsame Praxis
}

\author{
Clara Arnold und Samia Mohammed
}

\section{Warum Kritik in der Krise?}

Kritik scheint angesichts der Coronapandemie den Atem anhalten zu müssen. Das Coronavirus, das sich binnen Wochen am Anfang des Jahres 2020 pandemisch über den Globus ausbreitete, stürzte die Welt in multiple Krisen. Diese begegnen uns damals wie heute in drastischen Bildern und Meldungen und verweisen auf die Verwundbarkeit menschlicher Gesellschaften. Dabei ist beispielsweise an den Pflegenotstand zu denken, der sich in schlechter Bezahlung und Unterbesetzung in der Pflegebranche zeigt, oder an eine Vielzahl systemrelevanter Berufsgruppen, deren Beschäftigte zumeist prekär arbeiten und dabei Tätigkeiten nachgehen, bei denen die Einhaltung physischen Abstands nicht möglich ist. Einen Großteil der Kosten der Pandemie scheinen diejenigen tragen zu müssen, auf die wir in besonderem Maße angewiesen sind. Zugleich ist die Ungleichverteilung von Leid und Tod an der Tagesordnung, wenn z.B. in den USA Afroamerikaner*innen weit überproportional an Covid-19 erkranken und häufiger sterben als Weiße oder wenn an den europäischen Außengrenzen Geflüchtete in Lagern festgesetzt und von der Gesundheitsversorgung ausgeschlossen werden. Denn: Der staatliche Schutz von Leben bedeutet immer nur den Schutz von einigen und nicht von allen. Während diese Phänomene oft nicht prominent als Krisen gedeutet werden, zeigt die Proklamation der Pandemie als Krise, dass derartige Deutungen das Ergebnis von Prozessen des Ein- und Ausschlusses sowie politischer Machtkonstellationen sind. Dieser Umstand sowie die beschriebenen Verhältnisse waren und sind kritikwürdig. Sie präsentieren sich uns im Zuge der Pandemie auf eindringliche Art und Weise als ungerecht und ausbeuterisch und weisen so gerade auf die Notwendigkeit einer Kritik in der Krise hin. Kritische politische Theorie dient dabei als Voraussetzung und Mittel, um (Herrschafts-)Verhältnisse zu verstehen und zu verändern, und interveniert zugleich als Teil politischer Praxis.

Doch auch die Beschreibung und Kritik der pandemischen Situation selbst steht vor bis heute nicht gekannten Schwierigkeiten: So stellt die Pandemie eine existentielle Bedrohung menschlichen Lebens und Zusam- 
menlebens dar, weshalb nach unserem Eindruck im öffentlichen und wissenschaftlichen Diskurs die kritische Reflexion gegenüber der unmittelbaren Pandemiebekämpfung als nachrangig zu gelten scheint. Dieser Eindruck wird verstärkt durch eine in der Krise weiter zunehmende Hegemonialisierung faktenbasierter, am naturwissenschaftlichen Erkenntnismodell orientierter (Sozial-)Wissenschaft, die Formen kritischer politischer Erkenntnisproduktion zurückdrängt. Erschwert werden die Ausgangsbedingungen für Kritik zudem durch krude und antisemitische Verschwörungsmythen von Wissenschaftsgegner*innen, die sich teils sehr öffentlichkeitswirksam als maßgebliche Kritiker*innen an staatlichen Coronamaßnahmen zu präsentieren suchen. Eigene Kritik läuft dadurch Gefahr, als grundsätzliche Delegitimierung von Krisenbewältigungspolitiken missverstanden zu werden. Diese komplexe Gesamtkonstellation ergibt somit verschiedene Formen der Verschränkung von Kritik und Krise, die eine Kritik in der Krise geradezu herausfordern.

Wir möchten auf diese Herausforderung in und mit diesem Band durch drei spezifische Situierungen von Kritik in der Krise reagieren, die Ausgangs- und Produktionsbedingungen unserer Überlegungen darstellen. Erstens stellen wir fest, dass sich viele der analysierten Phänomene oder Eindrücke in den Wochen veränderten, in denen wir über sie schrieben. Die historische Bedingtheit von kritischer (Gesellschafts-)Theorie, wie sie in dieser Erfahrung zum Ausdruck kommt, ist aber nicht nur ein Problem, sondern vielmehr ein konstitutives Element ihrer selbst. Im Sinne der Kritischen Theorie und ihrer zentralen Einsicht, dass die Wahrheit selbst einen Zeitkern hat, stellt dies also kein wirkliches Hindernis dar: Vielmehr zeigt sich so, dass Kritik immer wieder aktualisiert werden muss und sich ebenso wie die gesellschaftlichen Verhältnisse - jedoch nicht unbedingt im Einklang mit ihnen - beständig verändert (vgl. Geuss 2019: 348). Wir situieren unsere Überlegungen also historisch in den ersten Monaten der Pandemie, was auch bedeutet, dass sie in erster Linie Entwicklungen und Phänomene dieser Zeit reflektieren und verhandeln.

Zweitens hat die Krise auch uns Autor*innen neu situiert und auf andere Plätze verwiesen. Denn sie hat vor unserem Alltag und unserem gemeinsamen Ort, in diesem Fall der Universität Bielefeld, keinen Halt gemacht. Zwischen Normalitätserhaltung und Ausnahmezustand wurde auch unser bisheriger Alltag als Studierende, Promovierende und Lehrende durcheinandergeworfen. Unser seit dem Sommersemester 2019 bestehendes Kolloquium zu kritischer politischer Theorie konnte plötzlich nicht mehr in gewohnter Form stattfinden und so gestalteten wir es zu einer digitalen Textwerkstatt um. Dass das anders werden würde, war uns von vornherein bewusst, denn seit Anbeginn waren unsere Treffen von lebhaften Diskussio- 
nen und dem persönlichen Kontakt in der Gruppe geprägt. Als uns klar wurde, dass dieser Austausch nicht vollständig ins Digitale übertragbar war, ohne enttäuscht zu werden, schien uns die gemeinsame Arbeit am Text als eine sinnvolle Alternative. Nicht nur das gemeinsame Interesse an kritischer politischer Theorie vereint uns, sondern auch die Erfahrung, dass die Beteiligung unterschiedlicher wissenschaftlicher Statusgruppen mit studentischen Perspektiven in der Mehrheit für das Diskutieren und Kommentieren nicht nur fruchtbar, sondern für uns mittlerweile auch unverzichtbar geworden ist. Diese Multiperspektivität prägt auch den vorliegenden Sammelband, der nicht zuletzt aufgrund unterschiedlicher Erfahrungen im wissenschaftlichen Betrieb eine diverse Sammlung von Überlegungen zur aktuellen Situation darstellt.

Und drittens ist dieses Projekt nicht im luftleeren Raum entstanden: Es ist als Ganzes situiert innerhalb neoliberaler Bedingungen der Wissensproduktion. So ist der Produktivitätsdrang und -zwang, der akademische Kreise nach ersten krisenbedingten Momenten der Schockstarre wieder mit voller Wucht erfasste, durchaus selbst einer Kritik zu unterziehen. Denn er steht unter neoliberalen Vorzeichen und trägt zu dem bei, was als Normalität oder Standard sogar noch im Ausnahmezustand gilt. Dabei muss immer auch berücksichtigt werden, wer in der Krise diesem Standard überhaupt gerecht werden kann. Gleichzeitig ist dieser Drang mitunter als Krisenbewältigungsmodus und Möglichkeit zu verstehen, sich denkend und schreibend zwar nicht der Illusion hinzugeben, der Krise irgendwie souverän gegenüberzustehen, so jedoch zumindest ein Stück Handlungsfähigkeit aus der Ohnmacht zurückzugewinnen. Darüber hinaus versuchen wir damit unserer selbstgewählten oder zugeschriebenen gesellschaftlichen Rolle als Wissenschaftler*innen gerecht zu werden und der Welt tätig beizuwohnen.

In dieser ambivalenten Situation hat uns das gemeinsame Buchprojekt in den vergangenen Monaten in vielerlei Hinsicht motiviert und beim Nachdenken unterstützt. In mehreren Runden haben wir in der gesamten Gruppe unsere ersten Textentwürfe in Peer-Review gelesen, kommentiert und diskutiert, uns angeregt und ermutigt, Ideen weiterzuverfolgen, unsere Argumente trotz eigener Unsicherheiten stark zu machen und im Licht der Coronakrise die Themen zu beleuchten, die uns am Herzen liegen oder durch die Krise besondere Dringlichkeit erlangt haben.

Daraus ist für uns die Möglichkeit entstanden, kritisches Denken als gemeinsame Praxis auch in der Krisensituation nicht aufzugeben. So stehen am Ende der Arbeit an diesem Sammelband und am Anfang neuer Diskussionen 13 Beiträge einer Kritik in der Krise, die wir im Folgenden thematisch systematisiert vorstellen. 


\section{Zum Aufbau des Bandes}

Mit den im Folgenden versammelten Beiträgen wird keine einheitliche Theorielinie oder -perspektive verfolgt - vielmehr ist es gerade die Multiplizität von Perspektiven, Zugängen und auch Modi des Schreibens, die den vorliegenden Band auszeichnen. Dennoch häufen sich die Motive von Ambivalenz und Vieldeutigkeit in der Beobachtung und Beschreibung der Krise. Das Aufgreifen von Phänomenen, diskursiven Anrufungen und Ereignissen erfolgt oftmals in Form von Befragungen und kritischen Infragestellungen. Dabei verbleiben einige Beiträge im Modus der Kritik, während andere sich auf die Suche nach Antworten auf die Krise begeben. Im Versuch einer Systematisierung folgt der Aufbau dieses Bandes vier unterschiedlichen Verschränkungen von Kritik und Krise, die im Folgenden einzeln vorgestellt werden.

(1) Den inhaltlichen Einstieg machen vier Texte, die sich mit Phänomenen auseinandersetzen, die erst durch die Coronapandemie in ihrer in den Beiträgen verhandelten Form zutage getreten oder für die Schreibenden relevant geworden sind. Erscheint uns die Krise einerseits als Zuspitzung bestehender Problematiken, fördert sie andererseits gleichzeitig ganz neue Fragen zutage, die so zu Fragen der Coronazeit werden und sich als historisch-spezifische Ausdrücke dieser Situation deuten lassen.

Der erste Beitrag des Bandes von Lea Caroline Jonas setzt sich mit der diskursiv vermittelten Vorstellung einer ,Rückkehr zur Normalität' auseinander. Dabei bezieht sich Jonas maßgeblich auf Brigitte Bargetz' kritische Theorie des Alltags und zeigt, dass Alltag - verstanden als kritischer Erkenntnisgegenstand - einen Zeit-Raum der gesellschaftlichen Entscheidung(en) verkörpert, der durch seinen nicht-dichotomisierenden Charakter das Potential für das Einfordern politischer Lösungen der Krise birgt.

Die Veränderungen unserer vermeintlichen Normalität durch die Maßnahmen der Pandemiebekämpfung werden unter anderem danach beurteilt, wie stark sie die individuelle Freiheit einschränken. Der Beitrag von Samia Mohammed stellt die Privilegierung eines primär negativen Freiheitsverständnisses im Kontext der Krisenbewältigung anhand von vier problematisierten Gesichtspunkten kritisch infrage: dem Ausblenden (kollektiver) Vulnerabilitäten; dem Rückgriff auf einen methodischen wie normativen Individualismus; der Vorstellung, dass wir mit der Rückkehr in einen vermeintlichen Normalzustand nach der Krise auch die Freiheit zurückgewinnen; sowie der Verschränkung negativer Freiheit mit dem Neoliberalismus.

Einige der einprägsamsten Formulierungen, die Eingriffen in den Alltag der Menschen durch Maßnahmen und Einschränkungen politisch Legiti- 
mation verschaffen, bedienen sich Kriegsmetaphorik. Deren Verwendung seitens der französischen, britischen, US-amerikanischen und - mit Abstrichen - der deutschen Regierung problematisiert Malte Pasler in seinem Essay, indem er zeigt, dass der Rückgriff auf Kriegsmetaphern zunächst zwar naheliegend erscheinen mag, jedoch u.a. einer autoritären Auffassung von Politik Vorschub leistet.

Andreas Vasilache beschließt diesen Abschnitt mit Überlegungen zum Immunitätsausweis, in dessen Einführung er ein Beispiel für die in der Coronakrise beobachtbare Spannung zwischen Legalität einerseits und exekutiver wie privatrechtlicher Faktizität andererseits erkennt. Dabei diskutiert er die auf den ersten Blick paradoxe Gleichzeitigkeit von legislativer Zurückweisung der Institutionalisierung eines Immunitätsausweises und der faktischen Etablierung unterschiedlicher Formen informeller Immunitätsausweise.

(2) Regierungsseitige Maßnahmen der Krisenbewältigung und des Bevölkerungsschutzes wurden schnell breitenwirksam als ,biopolitisch' gedeutet. Damit wurde häufig auch eine Kritik am Zugriff auf oder der Verwaltung von Körpern durch staatliche Institutionen intendiert. Doch welche Aussagekraft hat dieses Label im Kontext der Coronakrise überhaupt? Aus kritisch-politischer Perspektive drängt sich die Frage auf, wie wir in dieser ,Zeit der Exekutive' regiert werden - und das nicht nur von staatlich-souveräner Seite - und was daran aus welcher Perspektive zu problematisieren ist. Vier Beiträge unterziehen unter Zuhilfenahme politiktheoretischer Begriffsapparate (Ausnahmezustand - Biopolitik - Ideologie Demokratie) krisenpolitische Regierungstechniken einer kritischen Analyse.

Der erste Beitrag von Demokrat Ramadani versucht, die Coronapandemie als Ausnahmezustand zu deuten. Dabei arbeitet er mithilfe einer radikaldemokratischen Befragung drei Typen des Ausnahmezustandes heraus: den Ausnahmezustand gegen die Demokratie als Überführung einer demokratischen Ordnung in eine Diktatur, den Ausnahmezustand für die Demokratie als temporäre Modifikation demokratischer Institutionen und den Ausnahmezustand durch die Demokratie als Revitalisierung der Demokratie aus sich selbst heraus.

Simon Duncker zieht als Gegenkonzept zur pauschalen Subsumption staatlicher Pandemiepolitik unter den Begriff der Biopolitik ein differenzierteres Begriffsrepertoire heran, um die Coronamaßnahmen zu deuten. Er ordnet diese dabei gemäß ihrer Regierungsrationalitäten den Machtformen Foucaults zu und zielt so darauf ab, das Zusammenspiel der aufgegriffenen Regierungstechniken als Corona-Dispositiv zu beschreiben. 
Die beiden darauffolgenden Aufsätze dieses Abschnitts vertreten aus unterschiedlichen Perspektiven die Ansicht, dass die Coronapandemie bzw. die seither getroffenen Maßnahmen nur wenig überraschend sind.

Von dieser Beobachtung ausgehend arbeitet Kristoffer Klement heraus, dass die deutsche Coronapolitik als Ausdruck einer bewährten Krisenlogik verstanden werden kann, in der Angstideologien die Autorität einer funktionalen Schutzvernunft für politisches Handeln stärken. Diese untergräbt wiederum im demokratischen Gewand Ansprüche politischer Selbstregierung.

Gerrit Tiefenthal folgt in seinem Beitrag der noch grundlegenderen Annahme, dass der Ausbruch der Pandemie selbst keineswegs unerwartet geschah. Dass dennoch entsprechende Präventionsmaßnahmen nicht im nötigen Umfang getroffen wurden, sondern, im Gegenteil, die Bedingungen für eine solche Pandemie eher noch verbessert wurden, liegt seiner Argumentation zufolge daran, dass westliche Demokratien Pfadabhängigkeiten folgen, die sie als alternativlos präsentieren.

(3) Im dritten Teil des Bandes versammeln sich Beiträge, die allesamt die Krise als etwas beschreiben, das auf bereits länger bestehende Missstände hinweist. Dabei spitzt die Coronapandemie diese Krisen (national - international - global) besonders zu. Sie wirkt förmlich als Brennglas für gesellschaftliche und politische Verhältnisse, die in den Beiträgen unterschiedlichen Kritiken unterzogen werden.

Den Auftakt macht hier Nele Weiher, die das Verhältnis von Männlichkeit und Verschwörungsmythen im Kontext der Pandemie analysiert. Dabei zeigt sie, dass die diagnostizierte Krise der Männlichkeit, die mit multiplen, in der Krise verstärkten Verunsicherungserfahrungen einhergeht, das Suchen nach einfachen Erklärungen in Verschwörungen begünstigt.

Dass die Pandemie auch andernorts wütet, ist schon begrifflich evident - der Beitrag von Jamila Maldous führt uns dies am konkreten Beispiel Syriens vor Augen. Sie arbeitet mithilfe der Theorie Giorgio Agambens heraus, dass die Coronapandemie die Produktion nackten Lebens in dem von Kriegen gezeichneten Land dramatisiert und verschärft.

Auch die globale Perspektive, die Noab Marschner daran anschließend einnimmt, zeigt, dass bereits vor Einbruch der Coronakrise multiple andere Krisen auf der ganzen Welt herrschten. Marschner untersucht den spezifischen Zusammenhang von Corona- und Klimakrise und kritisiert sowohl die starre Gegenüberstellung als auch die einfache Gleichsetzung beider Krisen. Dem wird ein Appell für ein integratives Vorgehen gegen multiple sozial-ökologische Krisen entgegengestellt, das statt einer Rückkehr zur ,Normalität ${ }^{`}$ eine Systemtransformation erforderlich machen würde. 
(4) Der letzte und vierte Teil des Bandes nimmt die bereits thematisierte selbstreflexive Diagnose zum Ausgangspunkt, dass Kritik selbst im Zuge der Coronapandemie in einer Krise steckt.

Dabei fragt Oliver Flügel-Martinsen, ob das naturwissenschaftliche Erkenntnismodell in Zeiten der Pandemie alternativlos geworden ist. Entgegen diesem Eingangsverdacht meldet er bleibenden Bedarf an einer kritischen politischen Theorie der Pandemie an, die über das Paradigma harter Wissenschaftlichkeit hinausdenken muss, da es gerade jetzt starke Gründe dafür gibt, objektivitäts- und wahrheitsskeptisches Denken wachzuhalten.

Der letzte Beitrag des Bandes von Clara Arnold lenkt den Blick auf die Bedeutung von Präsenz im universitären Kontext. Die Erfahrung der körperlichen Abwesenheit der ersten Monate seit Beginn der Pandemie weist darauf hin, dass es dabei um mehr als die Frage der Digitalisierung von Hochschulen geht. So zeigt Arnold, dass Präsenz - verstanden als Weltverhältnis - Grundbedingung politischen Einspruchs ist.

\section{Literaturverzeichnis}

Geuss, Raymond. 2019. Normativität in der Kritischen Theorie der Politik. In: Ulf Bohmann/Paul Sörensen (Hrsg.), Kritische Theorie der Politik. Berlin: Suhrkamp, S. 348-363. 
